\title{
Fluctuating Hydrodynamics for Ionic Liquids
}

\author{
Konstantinos Lazaridis ${ }^{1}$, Logan Wickham ${ }^{2}$, Nikolaos Voulgarakis ${ }^{1, *}$ \\ ${ }^{1}$ Department of Mathematics and Statistics, Washington State University, Pullman, 99163 \\ ${ }^{2}$ Department of Computer Science, Washington State University, Richland, 99354
}

\begin{abstract}
We present a mean-field fluctuating hydrodynamics (FHD) method for studying the structural and transport properties of ionic liquids in bulk and near electrified surfaces. The free energy of the system consists of two competing terms: (1) a LandauLifshitz functional that models the spontaneous separation of the ionic groups, and (2) the standard mean-field electrostatic interaction between the ions in the liquid. The numerical approach used to solve the resulting FHD-Poisson equations is very efficient and models thermal fluctuations with remarkable accuracy. Such density fluctuations are sufficiently strong to excite the experimentally observed spontaneous formation of liquid nano-domains. Statistical analysis of our simulations provides quantitative information about the properties of ionic liquids, such as the mixing quality, stability, and the size of the nano-domains. Our model, thus, can be adequately parameterized by directly comparing our prediction with experimental measurements and all-atom simulations. Conclusively, this work can serve as a practical mathematical tool for testing various theories and designing more efficient mixtures of ionic liquids.
\end{abstract}

Keywords:

Mathematical modeling, mean field theory, fluctuating hydrodynamics, interfacial nanostructure, room temperature ionic liquids, electrical double layer.

\section{Introduction}

In the past decade, extensive studies have been conducted on room temperature ionic liquids (RTIL) mainly because of their intriguing physical properties as compared to the traditional electrolytes $[1,2]$. Its potential applications include high storage devices (super-capacitors) [3], batteries [4], lubrication of micro-electromechanical machines [1], dye-sensitized photoelectrochemical cells [5], and gating devices [2]. In a nutshell, RTIL can be considered as a new type of electrolyte due to their wide range of structural transitions near electrified surfaces [6, 7]. Experimental studies and atomistic-level simulations have reported overscreened and crowded aggregations of ions near electrodes that are accompanied by lamellar, bicontinuous, and spongelike layerings $[8,9,10,11,12]$. This structural morphology of RTIL can be tuned accordingly to increase the energy density and modify the transport properties of the electric double bilayer (EDL), i.e., the interface between the ionic liquid and a charged metallic surface [13].

Traditional mean-field studies for dilute electrolytes, such as the Gouy-ChapmanStern (GCS) theory [14], predict the formation of an exponentially decaying charged

*corresponding author: n.voulgarakis@wsu.edu

Preprint submitted to Physics Letters A

January 10, 2017

(C) 2016. This manuscript version is made available under the Elsevier user license 
layer near the metallic surface. Such behavior results in capacitance that is a monotonic function of the applied electric field. Recent experiments, however, have revealed a voltage-driven anomalous differential capacitance resulting from sudden changes in liquid morphology at the EDL [15]. This behavior has signatures of first-order phase transition and calls for theoretical studies beyond the traditional GCS approach. Modified mean-field models are capable of capturing screened and crowded interfacial effect that results in a non-monotonic voltage dependence of the capacitance [16, 2, 17]. However, they do not predict a first-order phase transition. Very recently, Limmer introduced a novel mean-field model that describes the experimentally observed anomalous behavior of the capacitance [18]. He particularly showed that this phenomenon results from the interplay of the short-range repulsion and long-range electrostatic interactions between the oppositely charged ionic groups. Using a Landau-Lifshitz-Poisson model, Gavish and Yochelis proceeded one step further and studied the interplay between the bulk nano-domains and the periodic layering at EDL [19]. Lee and Perkin followed a substantially different approach and showed that ion-image interaction might explain the anomalous transition in the differential capacitance [20]. They also mentioned that this behavior depends on the bulk energy of the RTIL.

The above mentioned theoretical approaches have qualitatively explained the mechanisms underlying the intriguing morphology of RTIL. However, these methods cannot provide quantitative results that could help design more efficient ionic liquids mixtures. The problem is simply too complicated to be solved analytically near electrified surfaces. Besides, deterministic numerical simulations cannot provide reliable predictions since they do not probe the entire free energy landscape of the system. To overcome this limitation, we present a stochastic mathematical model that directly compares with the experimental observations and all-atom molecular dynamics simulations. Our approach describes thermal noise through the standard FHD framework, which is a relatively well-developed method for modeling nanoscale fluid dynamics. Although initially introduced in an attempt to describe thermal fluctuations in homogeneous liquids [21], FHD has now become sophisticated enough to model mass, momentum, and energy transfer [22, 23, 24, 25, 26, 27, 28, 29] in multiphase-multicomponent fluid flows [30, 31] with or without reactive species $[32,33]$ for length scales as small scales as $5 \AA[24]$. FHD has also been incorporated in many popular fluid-structure methods to capture the effect of fluid thermal fluctuations on nanostructure dynamics $[34,35,36,37,38,39,24]$.

In this study, we present an FHD approach for ionic liquids designed to model not only the morphology but also the transport properties of the mixture. For this purpose, the governing equations of the system include the mass, component, and momentum balance equations coupled with the Poisson equation that provides the electrostatic potential in the system. To describe the spontaneous component separation, we consider a Landau-Lifshitz free energy functional similar to the Ref. [19] but appropriately modified to accommodate thermal fluctuations. The spatial gradients are approximated using the central difference staggerd scheme presented in Ref. [24], while the time integration of the governing equations is performed using a stochastic second order Runge-Kutta method [42]. The Poisson equation is solved simultaneously at each time step using a fast Fourier transform (FFT). Extensive numerical simulations proved that our approach can accurately reproduce the statistical fluctuations of the density fields and describe the experimentally observed nanostructuring in the bulk and near the EDL.We also show that the presence of thermal fluctuations 
may lead the system to ground states that are substantially different compared to the steady states of deterministic simulations of ionic liquids. Our approach, thus, can provide a quantitative analysis of RTIL and be a numerical framework for testing various theories and designing more efficient ionic liquids mixtures at the nanoscale.

The structure of the paper is as follows. In Sec. (2), we introduce the FHD equations for ionic liquids, and in Sec. (3), we present the numerical scheme used to solve the stochastic partial differential equations. In Sec. (4), we present the results of our numiercial simulations. Specifically, Sec. (4.1) is devoted to the dynamics of RTIL in the absence of an external electrostatic field. We first validate the accuracy of our numerical approach and then statistically analyze the fluctuations of the order parameters. In Sec. (4.2), we model an ionic liquid nanoconfined between two oppositely charged electrodes (nano-capacitor) and discuss the ability of our approach to reproduce the intriguing morphology of ionic liquids.Differences between stochastic and deterministic modeling of ionic liquids are also discussed. Finally, in Sec. (5), we briefly summarize and discuss our results.

\section{FHD equations for ionic liquids}

We have assumed a symmetric ionic liquid that consists of monovalent anions and cations of charge per unit mass $z_{+}=z_{0}$ and $z_{-}=-z_{0}$, respectively, where $z_{0} \in \mathbb{R}^{+}$. If $\rho_{+}$and $\rho_{-}$represents the mass density of the two opposite ions, we can define the total mass density of the system as $\rho=\rho_{+}+\rho_{-}$and the mass fraction difference as $c=c_{+}-c_{-}$, where $c_{+}=\rho_{+} / \rho$ and $c_{-}=\rho_{-} / \rho$. The total charge per mass unit is given by $z=z_{0} \rho c$. If the electrostatic potential of the system is denoted by $\phi$, and $\mathbf{x} \in \mathbb{R}^{\mathbf{3}}$ represents the spatial coordinates, the total mean field energy of the system can be written as

$$
F=F_{0}[c(\mathbf{x}), \rho(\mathbf{x})]+F_{e}[c(\mathbf{x}), \rho(\mathbf{x}), \phi(\mathbf{x})] .
$$

The first term in Eq. (1) represents the free energy of the system in the absence of electrostatic interaction and is given by a Ginzburg-Landau functional

$$
\left.F_{0}[c(\mathbf{x}), \rho(\mathbf{x})]=\int\left(\psi_{c}(c)+\frac{k_{c}}{2}|\nabla c|^{2}+\psi_{\rho}(\rho)+\frac{k_{\rho}}{2}|\nabla \rho|^{2}\right)\right) d \mathbf{x},
$$

where $\psi_{\rho}$ and $\psi_{c}$ are the local free energy densities for the order parameters and $\rho$ and $c$, respectively. Both local terms have a double well structure, i.e., $\psi_{0}(\rho)=A\left(\rho-\rho_{l}\right)^{2}(\rho+$ $\left.\rho_{v}\right)^{2}$, with minima at $\rho_{l}$ (liquid) and $\rho_{v}$ (vapor), and $\psi_{0}(c)=B(c-1)^{2}(c+1)^{2} / 2$ with two minima at -1 (cations) and +1 (anions). The coefficients $A$ and $B$ are a measure of the barrier heights. The mass density and mass fraction square gradients represent the free energy penalty for having phase and component interfaces, respectively, where $k_{\rho}$ and $k_{c}$ are the corresponding gradient coefficients.

The second term in Eq. (1) is the electrostatic energy of the system

$$
F_{e}[c(\mathbf{x}), \rho(\mathbf{x}), \phi(\mathbf{x})]=\int\left(z_{0} \rho c \phi-\frac{1}{2} \epsilon|\nabla \phi|^{2}\right) d \mathbf{x}
$$

where $\epsilon$ is the permittivity.

Under these assumptions, the mass, momentum and component balance equations are written as follows:

$$
\frac{\partial \rho}{\partial t}=-\nabla \cdot \mathbf{g}
$$




$$
\begin{gathered}
\frac{\partial \mathbf{g}}{\partial t}=\nabla \cdot(\mathbf{R}+\boldsymbol{\Pi}+\widetilde{\mathbf{\Pi}}) \\
\frac{\partial(\rho c)}{\partial t}+\nabla \cdot(\rho c v)=-\nabla \cdot(\mathbf{J}+\widetilde{\mathbf{J}}), \\
\nabla^{2} \phi=\frac{z \rho c}{\epsilon}
\end{gathered}
$$

where $t \in \mathbb{R}^{+}, \mathbf{g}=\rho \boldsymbol{v}$ is the momentum density, and $\boldsymbol{v}$ the velocity field of the fluid. The three tensors, $\mathbf{R}, \boldsymbol{\Pi}$ and $\widetilde{\boldsymbol{\Pi}}$, in the momentum balance equation (Eq. (5)), are the reversible, viscous, and fluctuating stress, respectively. Deriving the momentum balance equation through the least action principle shows that $\nabla \cdot \mathbf{R}=$ $-\rho(\delta F / \delta \rho)_{c}+(\delta F / \delta c)_{\rho} \nabla c$, which leads to

$$
\nabla \cdot \mathbf{R}=\nabla \cdot \mathbf{R}^{\rho}+\nabla \cdot \mathbf{R}^{c}-z_{0} \rho c \nabla \phi
$$

where

$$
\mathbf{R}^{\rho}=\left[-p_{0}-k_{\rho} \rho \nabla^{2} \rho+\frac{k_{\rho}|\nabla \rho|^{2}}{2}\right] \mathbf{I}-k_{\rho} \nabla \rho \otimes \nabla \rho
$$

and

$$
\mathbf{R}^{c}=\left[\psi_{0}(c)+\frac{k_{c}}{2}|\nabla c|^{2}\right] \mathbf{I}-k_{c} \nabla c \otimes \nabla c
$$

are the reversible stress tensors for the order parameters $\rho$ and $c$, respectively. In this work, we have assumed that there is no liquid-vapor phase separation and have neglected the mass density gradient. In Eq. (9), $p_{0}$ is the thermodynamics pressure, i.e., $p_{0}=\rho \partial \psi_{\rho} / \partial \rho-\psi_{\rho}[30]$. For simplicity, we have assumed that $\mathbf{R}_{\rho}$ is the hydrodynamic pressure of the system

$$
\mathbf{R}^{\rho} \simeq c_{T}^{2} \rho \mathbf{I}
$$

where $c_{T}$ is the sound velocity of the mixture. We also have assumed that the velocity fluctuations are small enough to neglected the nonlinear advection term in Eq. (5). The Newtonian dissipative stress in momentum balance equation is expressed as

$$
\boldsymbol{\Pi}=\eta_{S}\left(\nabla \boldsymbol{v}+\nabla \boldsymbol{v}^{T}\right)+\left(\eta_{B}-\frac{2}{3} \eta_{S}\right)(\nabla \cdot \boldsymbol{v})
$$

where $\eta_{S}$ and $\eta_{B}$ are the shear and bulk viscosity, respectively. For simplicity, we assume that the viscosity coefficients have the same values for both ionic groups. The fluctuating tensor $\widetilde{\boldsymbol{\Pi}}$ in Eq. (5) is treated as a white noise with covariances determined by the fluctuation dissipation theorem [40, 41], i.e.,

$$
\begin{aligned}
\left\langle\widetilde{\Pi}^{\alpha \beta}(\mathbf{x}, \mathbf{t}) \cdot \widetilde{\Pi}^{\gamma \delta}\left(\mathbf{x}^{\prime}, \mathbf{t}^{\prime}\right)\right\rangle & =2 k_{B} T\left[\eta_{S}\left(\delta_{\alpha \gamma} \delta_{\beta \delta}+\delta_{\alpha \delta} \delta_{\beta \gamma}\right)+\left(\eta_{B}-\frac{2}{3} \eta_{S}\right) \delta_{\alpha \beta} \delta_{\gamma \delta}\right] \\
& \times \delta\left(t-t^{\prime}\right) \delta\left(\mathbf{x}-\mathbf{x}^{\prime}\right)
\end{aligned}
$$

where $k_{B}$ is Boltzmann's constant, $T$ is the temperature of the system, and alpha, beta, $\gamma$, and $\delta=x, y$, or $z$.

In the component balance Eq. (6) $\mathbf{J}=\mathbf{J}_{\mathbf{2}}-\mathbf{J}_{\mathbf{1}}=\mathbf{2} \mathbf{J}_{\mathbf{2}}$ represents the mass flux difference and $\widetilde{\mathbf{J}}$ is the corresponding fluctuating flux. The diffusive flux is given by $\mathbf{J}=$ 
$\lambda \nabla \mu$, where $\lambda$ is the mobility of the mixture, and $\mu$ is the generalized electrochemical potential difference

$$
\mu=\frac{1}{\rho}\left(\frac{\delta F}{\delta c}\right)_{\rho}=\frac{1}{\rho}\left(\frac{\partial \psi_{0}}{\partial c}-\nabla \cdot\left(k_{c} \nabla c\right)\right)+z \phi .
$$

The first term in Eq. (14) represents the chemical potential difference and the second term is the electrostatic contribution. The fluctuating component mass flux $\widetilde{\mathbf{J}}$ is given by a Gaussian white noise with zero mean and covariance $[40,41]$

$$
\left\langle\widetilde{J}^{\alpha}(\mathbf{x}, t) \cdot \widetilde{J}^{\beta}\left(\mathbf{x}^{\prime}, t^{\prime}\right)\right\rangle=2 \lambda k_{B} T \delta_{\alpha \beta} \delta\left(t-t^{\prime}\right) \delta\left(\mathbf{x}-\mathbf{x}^{\prime}\right) .
$$

Equation (7) is simply the Poisson equation, which can be derived by assuming that the electrostatic potential $\phi$ is a critical point of the action.

\section{Numerical Scheme}

To numerically solve the governing equations in two dimensions, we implemented the staggered discretization scheme presented in Ref. [24]. Specifically, we considered a rectangular domain $\Omega=\left(0, L_{x}\right) \times\left(0, L_{y}\right)$ bounded by $\partial \Omega$. The domain is discretized in $N_{x} \times N_{y}$ rectangular cells of sides $d_{x}=\mathrm{E}_{x} / N_{x}$ and $d_{y}=\mathrm{E}_{y} / N_{y}$. The centers of the cells are labeled by the index vector $(i, j)$, and the sides along $x$-axis and $y$-axis are labeled by $(i+1 / 2, j)$ and $(i, j+1 / 2)$, respectively, where $i=1, \ldots, N_{x}$ and $j=1, \ldots, N_{y}$. The corner indexes are denoted by $(i+1 / 2, j+1 / 2)$. By using the the finite difference method to approximate the spatial gradients the discretized governing equations can be written as:

$$
\begin{gathered}
\frac{\partial \rho_{i, j}}{\partial t}=-\frac{1}{d}\left[\left(g_{i+1 / 2, j}^{x}-g_{i-1 / 2, j}^{x}\right)+\left(g_{i, j+1 / 2}^{y}-g_{i, j-1 / 2}^{y}\right)\right] \\
\left.\frac{\partial g_{i+1 / 2, j}^{x}}{\partial t}=\frac{1}{d}\left\{\left[R_{i+1, j}^{\rho}-R_{i, j}^{\rho}\right)\right]+\left[R_{i+1, j}^{c}-R_{i, j}^{c}\right)\right]+ \\
{\left[\left(\Pi_{i+1, j}^{x x}-\Pi_{i, j}^{x x}\right)+\left(\Pi_{i+1 / 2, j+1 / 2}^{x y}-\Pi_{i+1 / 2, j-1 / 2}^{x y}\right)\right]+} \\
{\left[\left(\widetilde{\Pi}_{i+1, j}^{x x}-\widetilde{\Pi}_{i, j}^{x x}\right)+\left(\widetilde{\Pi}_{i+1 / 2, j+1 / 2}^{x y}-\widetilde{\Pi}_{i+1 / 2, j-1 / 2}^{x y}\right)\right]+} \\
\left.\left[z_{0}(\rho c)_{i+1 / 2, j}^{x}\left(\phi_{i+1, j}-\phi_{i, j}\right)\right]\right\} \\
\frac{\partial(\rho c)_{i, j}}{\partial t}=-\frac{1}{d}\left\{\left[\left((g c)_{i+1 / 2, j}^{x}-(g c)_{i-1 / 2, j}^{x}\right)+\left((g c)_{i, j-1 / 2}^{y}-(g c)_{i, j+1 / 2}^{y}\right)\right]+\right. \\
{\left[\left(J_{i+1 / 2, j}^{x}-J_{i-1 / 2, j}^{x}\right)+\left(J_{i, j-1 / 2}^{y}-J_{i, j+1 / 2}^{y}\right)\right]+} \\
\left.\left[\left(\widetilde{J}_{i+1 / 2, j}^{x}-\widetilde{J}_{i-1 / 2, j}^{x}\right)+\left(\widetilde{J}_{i, j-1 / 2}^{y}-\widetilde{J}_{i, j+1 / 2}^{y}\right)\right]\right\} \\
\frac{1}{d^{2}}\left[\left(\phi_{i-1, j}-2 \phi_{i, j}+\phi_{i+1, j}\right)+\left(\phi_{i, j-1}-2 \phi_{i, j}+\phi_{i, j+1}\right)\right]=z_{0}(\rho c)_{i, j} / \epsilon
\end{gathered}
$$

where

$$
\left\langle\widetilde{\Pi}_{i j}^{\alpha \beta}(t) \widetilde{\Pi}_{i^{\prime} j^{\prime}}^{\gamma \delta}\left(t^{\prime}\right)\right\rangle=\frac{2 k_{B} T}{A_{C}}\left[\eta_{S}\left(\delta_{\alpha \gamma} \delta_{\beta \delta}+\delta_{\alpha \delta} \delta_{\beta \gamma}\right)+\left(\eta_{B}-\frac{2}{3} \eta_{S}\right) \delta_{\alpha \beta} \delta_{\gamma \delta}\right] \delta_{i i^{\prime}} \delta_{j j^{\prime}} \delta\left(t-t^{\prime}\right),
$$


and

$$
\left\langle\widetilde{J}_{i j}^{\alpha}(t) \cdot \widetilde{J}_{i^{\prime} j^{\prime}}^{\beta}\left(t^{\prime}\right)\right\rangle=\frac{2 \lambda k_{B} T}{A_{C}} \delta_{\alpha \beta} \delta_{i i^{\prime}} \delta_{j j^{\prime}} \delta\left(t-t^{\prime}\right) .
$$

In Eqs. (20) and (21), $A_{c}$ is the surface area of each elementary cell, i.e., $A_{c}=$ $d_{x} d_{y}$. Equation (17) describes the momentum balance only in $x$-direction; however, the equation of the $g_{i, j+1 / 2}^{y}$ component can be derived following a similar approach. Although $\rho, c$, and $\rho c$ are computed at the center of each cell, we can estimate their values at the sides by linear interpolation, i.e., in $x$-direction, for instance, $(\rho c)_{i+1 / 2, j}^{x}=$ $\left[(\rho c)_{i, j}+(\rho c)_{i+1, j}\right] / 2$.

The discretized Eqs. (16)-(18) are integrated in time using a second order RungeKutta method [42], and the discrete Poisson Eq. (19) is solved at each time step using fast Fourier transform (FFT) [43].

\section{Results and discussion}

Our primary goal is to study the interplay between short and long range interaction and its effect on ionic liquid morphology. It is convenient, thus, to analyze the system regarding a control parameter that describes the ratio of the two interactions. To be consisntent with the work of Ref. [19], we choose the control parameter to be $\sigma=k_{c} / k_{B} T \lambda^{2}$, where $\lambda=\sqrt{\epsilon k_{B} T / z_{0}^{2} \rho_{0}^{2}}$. In this work, we keep the interfacial energy constant and vary the charge per mass density of the system, i.e., increasing $\sigma$ corresponds to increasing electrostatic energy. The rest of the model parameters remain constant. Specifically, the shear and bulk viscosity are $\eta_{S}=600 \mathrm{amu} / \mathrm{ps} \AA$ and $\eta_{B}=600 \mathrm{amu} / \mathrm{ps} \AA$, respectively. The strength of the double well is $A=0.33 k_{B} T$, and the sound velocity of the mixture is $c_{T}=100 \AA / \mathrm{ps}$. The mass density of the ionic groups is the same, i.e., $\rho_{1}=\rho_{2}=\rho_{0}$, where $\rho_{0}=1.2 \mathrm{amu} / \AA^{2}$. The temperature of the system is $T=300 \mathrm{~K}$, and the integration time step is $d t=0.005 \mathrm{ps}$.

One of the purpose of this paper is to show the importance of thermal fluctuations in simulations of ionic liquids. An appropriate discretization length must be small enough to ensure the presence of strong stochastic fluxes (see Eqs. (20) and (21)), but not smaller than a typical size of an atom. In this work, the size of the simulation box and the partition are so chosen that the discretization length is $d=5 \AA$. As shown in our previous works (see Refs. [24, 26]), if the finite size of the atoms is taken into consideration, FHD can perfectly reproduce the mass and momentum fluctuations of all-atom simulations even for length scales as small as $5-6 \AA$. However, the description of the transport properties of liquids, such as shear viscosity, breaks down at wavelengths of about $32 \AA$. However, in Refs. [28, 29], we showed that incorporation of a simple Maxwell model of viscoelasticity into the FHD framework can adequetly describe the transport properties of both Newtonian and complex fluids at scales as small as $12 \AA$. In this work, we focus only on the mass and momentum thermal fluctuations, as well as the long wavelength transport properties of ionic liquds. The incorporation of viscoelastic effects in our model, i.e., Non-Markovian FHD, will be presented elsewhere [44].

\subsection{Simulations in absence of external electrostatic field}

The first step is to check the accuracy and capabilities of our numerical scheme. For this purpose, we present the results of an ionic liquid in a square domain $L \times L$ discretized in $N \times N$ cells, where $L=128 \AA$ and $N=128$. We used periodic boundary 
conditions on $\partial \Omega$, and for initial condition, we considered that the ions are perfectly mixed and the temperature of the system is zero, i.e.,

$$
[\mathbf{g}(\mathbf{x}, 0), \rho(\mathbf{x}, 0), c(\mathbf{x}, 0)]=\left[\mathbf{0}, \rho_{0}, 0\right], \mathbf{x} \in \Omega .
$$

\subsubsection{Equilibration procedure}

In the absence of electrostatic interactions, i.e., $\sigma=0$, the ground state of the system consists of two well-separated and well-aligned (lamellar) stripes. One stripe corresponds to $c<0$ and the other to $c>0$. For increasing $\sigma$, the system tends to form more lamellar stripes in order to reduce the electrostatic energy of the system. At significantly high values of the $\sigma$, electrostatic interaction destroys this periodic morphology and creates randomized patterns of positive and negative total mass fraction difference.

Once the simulation is started, the total temperature of the system increases very fast from zero to the desired value of $T=300 \mathrm{~K}$. This process is very fast and takes approximately $2 \mathrm{ps}$, i.e., $t_{T} \approx 2$ ps. However, depending on the values of the system parameters, the energy of the ionic liquid reaches a plateau at a different time $t_{F}$ (see Fig. 1a). As shown in the inset of Fig. 1a, $t_{F}$ increases with decreasing $\sigma$. For very low values of the control parameter, the system approaches the ground state very slowly and it is practically impossible for the computer facilities used in this work to find the ground state of the system. It has to be mentioned that $t_{F}>t_{T}$ in all the simulations performed in this work. The equilibration time of the system can be defined as $t_{e q}=t_{F}+5 \mathrm{~ns}$. The extra $5 \mathrm{~ns}$ were added to ensure the system is perfectly in equilibrium.

Figures $1 \mathrm{~b}$-d show the density plot of the velocity field in the $x$-direction, the mass density and the mass fraction difference of the mixture, respectively, 5 ns after the equilibration time. As expected, the velocity and mass density fluctuate around their equilibrium values, $v_{0}=0$ and $\rho_{0}=1.25 \mathrm{amu} / \AA^{2}$. Figure $1 \mathrm{~d}$ illustrates the spontaneous component separation described by our mean-field FHD-Poisson approach. The detailed statistical analysis of Fig. 1 can provide useful information about the accuracy and capabilities of our method.

All results presented in this work were obtained by averaging 100 independent FHD simulations. The statistical sampling starts at $t=t_{e q}$, is obtained every $0.1 \mathrm{ps,}$ and lasts for 5 ns.

\subsubsection{Accuracy of the numerical scheme}

To test the accuracy of our numerical scheme, we directly compared the prediction of FHD simulations to the available statistical formulas. The probability density function (PDF) of $\sqrt{\rho^{x}} v^{x}$, for instance, must follow the Maxwell-Boltzman distribution

$$
P\left(\sqrt{\rho^{x}} v^{x}\right) \sim \exp \left(-\frac{A_{c}}{2 k_{B} T}\left(\sqrt{\rho^{x}} v^{x}\right)^{2}\right) .
$$

The PDF of the mass density fluctuations, as a different example, also has to be Gaussian [21]:

$$
P(\rho) \sim \exp \left(-\frac{A_{c} c_{T}^{2}}{2\langle\rho\rangle k_{B} T}(\rho-\langle\rho\rangle)^{2}\right),
$$

where $\langle\ldots\rangle$ represents the statistical average. 
In Fig. 2, we present the fitting of Eq. (22) to the numerical probability density function of $\sqrt{\rho^{x}} v^{x}$. The obtained standard deviation corresponds to a temperature $T^{\text {num }}=300.6 \mathrm{~K}$ with a relative difference of $0.2 \%$ as compared to the input temperature $(T=300 \mathrm{~K})$. A similar fit of Eq. (23) to the PDF of $\rho$ gives sound velocity $\left(c_{T}^{n u m}=100.16 \AA / \mathrm{ps}\right)$ that is very close to the input value, with a relative difference of $0.16 \%$. However, we have to mention that the compresibility of the system can be affected by the interfacial energy. Generally speaking, this can be observed in a system of high surface tension and many coexisting interfaces between the two ionic groups. This situation was not observed in the range of parameters used in this work. The relative error for temperature and sound velocity was persistently less than $1 \%$ in all numerical simulations performed here. According to Eq. 16, the total density $(\rho)$ of the system is conserved. The entire set of simulations performed in this worked showed that the combined staggered discretization scheme and the second order RK method conserve $\rho$ to the machines precision.

The successful fitting of Eqs. (22),(23) indicates that our numerical scheme accurately resolves the density fluctuations of ionic liquids at the nanosecond regime.

\subsubsection{Statistical analysis of mixture morphology}

Having verified the accuracy of our numerical approach, we move forward to studying the potential of our simulations in reproducing experimental measurements. Our main goal is to show how the liquid morphology depends on the control parameter $\sigma$. The most appropriate approach to answering this question is the statistical analysis of the mass fraction fluctuations. In Fig. 3a-c, we present density plots of $c$ for three different values of $\sigma$. It can be seen that for small $\sigma$ (Fig. 3a), where short range interfacial interactions dominate electrostatic interactions, the system tends to create well-aligned stripes. Increasing values of sigma simply decreases the width of the stripes or, equivelantly, increases the number of stripes. For $\sigma \gtrsim 5$, where electrostatic interactions start dominating over the short-range interactions, the system creates randomized very narrow islands of positive and negative $c$ to minimize the electrostatic energy (see Fig. 3b for $\sigma=7$ ). Note that for very high values of the control parameter (see Fig. 3c for $\sigma=23.1$ ), electrostatic interaction completely dominates the system and the ions are practically perfectly mixed, i.e., $c(\mathbf{x}) \approx 0$ for all $\mathbf{x} \in \Omega$.

There are two computations that can further analyze the density plots presented in Fig. 3a-c. The first and more direct one is the probability density function of $c, P(c)$. The $\mathcal{F}(c)=-k_{B} T \ln (P(c))$ provides the free energy of the system as a function of the order parameter $c$. In Fig. 3d, we present $\mathcal{F}(c)$ for $\sigma=7$. It can be seen that the free energy has a double well structure with two minima located at $c_{-}^{\min }$ and $c_{+}^{\min }$, separated by an energy barrier $\Delta \mathcal{F}$. The difference $\Delta c=c_{+}^{\min }-c_{-}^{\min }$ can provide an estimate of the mixing quality of the ionic groups: i.e., $\Delta c=2$ corresponds to perfect segregation and $\Delta c=0$ to perfect mixing. The value of $\Delta \mathcal{F}$ presents an estimate of the stability of the spontaneously generated patterns. In Fig. 3e, we plot $\Delta \mathcal{F}$ as a function of $\sigma$ and show that increasing $\sigma$ destabilizes the liquid patterns and favors mixing. There is a critical value of the control parameter, $\sigma^{*} \approx 10$, where the system is perfectly mixed, i.e., $\Delta c=0$ and $\Delta \mathcal{F}=0$. Note that increasing the sampling time does not result to visible improvement of the statistical averages. For example, doubling the sampling time for $\sigma=4.3$ showed a $10^{-3}$ relative change in the calculated free energy barrier presented in $3 \mathrm{e}$.

A second measurement that can provide information for the morphology is the 
power spectral density of $c$, for all permitted wave number vectors $\mathbf{q}=2 \pi(i, j) / L$, where $i, j=1,2 \ldots, N$. In Fig. 3f, we plot the power spectra as a function of $\mathbf{q}=$ $2 \pi(i, 0) / L$ for three different values of $\sigma$. The location of the central peak provides an estimate of the patterns periodicity, and consequently the width of the stripes, while the intensity of the peak is another way to estimate the stability of the patterns. We see that for small $\sigma$, a clear dominant peak is observed at relatively low $q$ (great stripes width) while increasing $\sigma$ shifts the power spectra to higher wave numbers (lower stripes width). The intensity of the signal also decreases monotonically with increasing $\sigma$. At even higher values of the control parameter, the signal disappears, indicating that the mixture has become uniform, i.e., the width and the lifetime of the stripes are zero. Here, we have to underline that the power spectra analysis can directly be compared to X-ray, neutron diffraction, and nonlinear spectroscopy [45].

The fact that we can model the statistical behavior of the system makes our approach more realistic and directly comparable with the experiments. Our FHD framework for ionic liquids can, thus, provide a robust mathematical tool for analyzing experiments of ionic liquids.

\subsection{Simulations under external electrostatic field}

Here, we study the morphology of the mixture in between two oppositely charged parallel electrodes. To simplify our simulations, we have assumed that the two electrodes are immersed in our two dimensional domain as shown in Fig. (4). Let us denote the charge per unit mass of each electrode by $z_{ \pm}^{e}= \pm z_{0}^{e}$, where $z_{0}^{e} \in \mathbb{R}^{+}$, the domains they occupy by $\Omega_{ \pm}^{e}$, and the boundaries of the electrodes with the ionic liquid by $\partial \Omega_{ \pm}^{e}$. For simplicity we have assumed that $z_{0}^{e}=z_{0}$. Note that increasing $z_{0}^{e}$ effectivily increases the voltage difference of the capacitor. The distance between the two electrodes is $D=100 \AA$. During the simulations, the electrodes are treated as a solid structure, i.e.,

$$
[\mathbf{g}(\mathbf{x}, t), \rho(\mathbf{x}, t), c(\mathbf{x}, t)]=\left[\mathbf{0}, \rho_{0}, \pm 1\right], \mathbf{x} \in \Omega_{ \pm}^{e} \text { and } t \in \mathbb{R}^{+}
$$

We use periodic boundary conditions on $\partial \Omega$, and no flux boundary conditions on $\partial \Omega_{ \pm}^{e}$, i.e.,

$$
\begin{gathered}
\mathbf{n} \cdot[\mathbf{J}+\tilde{\mathbf{J}}]=0 \\
\mathbf{n} \cdot[\mathbf{R}+\boldsymbol{\Pi}+\tilde{\boldsymbol{\Pi}}]=0,
\end{gathered}
$$

where $\mathbf{n}$ is the unit vector perpendicular to the electrodes. The implementation of periodic boundary conditions on $\partial \Omega$ allows us to still use a simple FFT approach to solve the Poisson Eq. (19). The initial conditions are similar to the previous section, that is, the two components are perfectly mixed and the temperature is zero:

$$
[\mathbf{g}(\mathbf{x}, 0), \rho(\mathbf{x}, 0), c(\mathbf{x}, 0)]=\left[\mathbf{0}, \rho_{0}, 0\right], \mathbf{x} \in \Omega \backslash \Omega_{ \pm}^{e} .
$$

Our solver computes the electrostatic potential in the entire $\Omega$ and the density fields $\rho, c$, and $\mathbf{g}$ in $\Omega \backslash \Omega_{ \pm}^{e}$. However, since we are interested in the behavior of the confined part of the ionic liquid we will present and discuss results only in the area between the two electrodes.

The deterministic simulations reported by Gavish and Yochelis in Ref. [19] can be reproduced by our model if thermal fluctuations are neglected, i.e., $T=0$. Similar to their work, the system variables have to be slightly randomized at $t=0$ in order to 
drive the system away from the metastable initial condition of Eq. (24). In Figs. 5 a-d show the steady state of the system for four different values of the control parameter. Similar to observation in Ref. [19], the system exhibits labyrinthine patterns for small values of $\sigma$ (see Fig. 5a for $\sigma=0.9$ ). For higher values of the control parameter, the system transitions from mixed labyrinthine -lamellar patterns (see Fig. 5b for $\sigma=8.3$ ) to pure lamellar morphology (see Fig. 5c for $\sigma=9.8$ ). According to Gavish and Yochelis [19], the presence of labyrinthine patterns is a consequence of a finite wavenumber instability. For considerably high values of the control parameter, the system is practically over-screened (see Fig. $5 \mathrm{~d}$ for $\sigma=21.1$ ), i.e., the charged layer next to the electrode completely balances the electrostatic potential of the electrodes and drives the remaining system to a neutral charge state $(c \approx 0)$.

The results of the deterministic simulations presented in Fig 5 a-d are perfectly valid at large length scales, where thermal fluctuations are negligible. However, at small length scales, strong thermal fluctuations can significantly change this scenario. The continuous presence of stochastic forces can drive the ionic liquid over local energy barriers to reach the global ground state of the system. Similar to Sec. 4.1, we expect that for low values of $\sigma$, the system forms well-aligned stripes, whereas for high values of $\sigma$, the system should result in randomized (labyrinthine) patterns. This behavior can indeed be observed in Figs. 5 e-h, which present time snapshots of the mass fraction difference at $T=300 \mathrm{~K}$ for four different values of the control parameter. Figs. $5 \mathrm{i}-\mathrm{l}$ show the corresponding spatial average of $c$ over $x$-direction, i.e., $\bar{c}_{y} \equiv \bar{c}_{j}=\sum_{i=1}^{N}\left\langle c_{i, j}\right\rangle / N$. The behavior of the system can be practically divided into four distinct regions. In region I, $0<\sigma \lesssim 1.0$, the ground state of the system is described by lamellar layering (see Fig. 5 e, for instance, for for $\sigma=0.9$ ). Increasing $\sigma$ from 0 to approximately 1 simply reduces the width of the stripes, or equivalently, increases the number of the stripes. In region II, $1 \lesssim \sigma \lesssim 6$, the free energy barrier of the system (see, Fig. $5 \sigma=X x$ ) becomes comparable to $k_{B} T$ and thermal fluctuations can temporarily destroy the lamellar morphology. This behavior is more evident in the middle of the capacitor, where the electrostatic potential of the electrodes is weaker (see Fig. 5f, for instance, for $\sigma=5.8$.) The resulting morphology is a mixed lamellar (close to the electrodes) and labyrinthine-type (in the bulk) of patterning. The corresponding behavior of $\bar{c}_{y}$ reveals a decaying periodic layering from region I to region II (see Fig. 5 $\mathrm{i}$ and $\mathrm{j}$ ). In region III, $6 \lesssim \sigma \lesssim 10$, the free energy barrier is significantly smaller than $k_{B} T$ and the lamellar layering is completely destroyed, leading to a labyrinthine type of patterns (see Fig. $5 \mathrm{~g} \sigma=9.8$ ). The corresponding behavior of $\bar{c}_{y}$ reveals the socalled crowded behavior (Fig. 5k). Such states consist of a dominantly charged layer next to the oppositely charged electrode. However, this layer is not strong enough to screen out the electrostatic potential of the electrodes $[6,2]$ and one or two more layers of decreasing charge might appear consecutively. In region IV, $\sigma \gtrsim 10$, the system is totally over-screened (see Fig. $5 \mathrm{~h}$ and $\mathrm{l}$ for $\sigma=21.1$ ).

All four regions detected by our FHD approach have been observed experimentally [7]. Atomic force microscopic experiments (AFM) can sense the pattern formation and report it in force-distance oscillating profiles [46]. The spatial periodicity of these patterns can also be experimentally detected by X-ray, neutron diffraction, and nonlinear spectroscopy studies [7]. Our numerical simulations can report results that can directly compare with these experiments. Specifically, $\bar{c}_{y}$ can be used to interpret AFM measurements, and the power spectra of $\bar{c}_{y}$ can be used to analyze the spectroscopy experiments. 


\section{Conclusions}

We have presented an FHD-Poisson framework for ionic liquids in bulk and near electrified metallic surfaces. Our approach describes both structural and transport properties of ionic groups through the coupled mass, component, and momentum balance equations. The free energy of the system consists of short-range interactions given by a Landau-Lifshitz functional that describe spontaneous phase separation, and a long-term interaction due to the electrostatic charge of the ionic liquids. Thermal fluctuations are incorporated in the governing equations through the FHD framework.

The numerical scheme for solving the stochastic partial differential equations is a combination of a) a central-differences staggered discretization scheme for the spatial gradients, b) a second order stochastic Runge-Kutta method for the temporal integration of the equations, and c) an FFT method for solving the Poisson equation. Direct comparison of our numerical simulations with available statistical formulas showed that our numerical scheme is remarkably accurate in describing the thermal fluctuations the system.

The stochastic nature of our system allows us to parametrize the governing equation by directly comparing our simulations with experimental observations and atomistic simulations. The numerical PDF of the mass fraction difference, for instance, can provide the free energy landscape of the ionic mixture. The obtained free energy profile (See Fig. 3e) can be used to compute the mixing quality of the ions as well as the stability of the spontaneously formed nano-domains. Besides, the power spectra of $c$, can be utilized to estimate the size of the nano-domains (see Fig. 3f). The morphology of a symmetric ionic liquid confined in a nano-capacitor was also studied. We showed that our approach can retrieve all the different bulk and near the electrode nanostructuring that has been observed experimentally. Specifically, we showed that increasing values of the control parameter, $\sigma$, can excite a) pure lamellar throughout the nanocapacitor, b) a lamellar layering near EDL accompanied by a labyrinhtine type of patterns in the bulk, c) pure labyrinhtine-type of layering (i.e., crowded state), and d) a single over-screened layer close to the electrodes (overscreened state).

Our FHD-Poisson approach, appropriately parametrized, can be a useful mathematical tool for testing and interpreting various proposed mechanisms for the intriguing physical properties of ionic liquids.

\section{Acknowledgments}

We thank the anonymous referee for his/her helpful comments. This material is based upon work supported by the National Science Foundation under Grant No. DMS 1418962.

\section{References}

[1] Anthony Somers, Patrick Howlett, Douglas MacFarlane, and Maria Forsyth. A Review of Ionic Liquid Lubricants. Lubricants 2013, Vol. 1, Pages 3-21, 1(1): 3-21, January 2013.

[2] Maxim V Fedorov and Alexei A Kornyshev. Ionic Liquids at Electrified Interfaces. Chem. Rev., 114(5):2978-3036, March 2014. 
[3] A Brandt, S Pohlmann, A Varzi, A Balducci, and S Passerini. Ionic liquids in supercapacitors. MRS Bulletin, 38(7):554-559, July 2013.

[4] Andrzej Lewandowski and Agnieszka Świderska-Mocek. Ionic liquids as electrolytes for Li-ion batteries-An overview of electrochemical studies. Journal of Power Sources, 194(2):601-609, December 2009.

[5] Qinghua Li, Qunwei Tang, Benlin He, and Peizhi Yang. Full-ionic liquid gel electrolytes: Enhanced photovoltaic performances in dye-sensitized solar cells. Journal of Power Sources, 264:83-91, October 2014.

[6] Susan Perkin. Ionic liquids in confined geometries. Phys. Chem. Chem. Phys., 14 (15):5052-5062, March 2012.

[7] Robert Hayes, Gregory G Warr, and Rob Atkin. Structure and Nanostructure in Ionic Liquids. Chem. Rev., 115(13):6357-6426, June 2015.

[8] Yu Zhuan Su, Yong Chun Fu, Jia Wei Yan, Zhao Bin Chen, and Bing Wei Mao. Double Layer of $\mathrm{Au}(100) /$ Ionic Liquid Interface and Its Stability in ImidazoliumBased Ionic Liquids. Angewandte Chemie, 121(28):5250-5253, June 2009.

[9] Yun-Xin Zhong, Jia Wei Yan, Mian-Gang Li, Xiao Zhang, Ding-Wen He, and Bing Wei Mao. Resolving Fine Structures of the Electric Double Layer of Electrochemical Interfaces in Ionic Liquids with an AFM Tip Modification Strategy. J. Am. Chem. Soc., 136(42):14682-14685, October 2014.

[10] Ahmet Uysal, Hua Zhou, Guang Feng, Sang Soo Lee, Song Li, Paul Fenter, Peter T Cummings, Pasquale F Fulvio, Sheng Dai, John K McDonough, and Yury Gogotsi. Structural Origins of Potential Dependent Hysteresis at the Electrified Graphene/Ionic Liquid Interface. J. Phys. Chem. C, 118(1):569-574, January 2014.

[11] Markus Mezger, Heiko Schröder, Harald Reichert, Sebastian Schramm, John S Okasinski, Sebastian Schöder, Veijo Honkimäki, Moshe Deutsch, Benjamin M Ocko, John Ralston, Michael Rohwerder, Martin Stratmann, and Helmut Dosch. Molecular Layering of Fluorinated Ionic Liquids at a Charged Sapphire (0001) Surface. Science, 322(5900):424-428, October 2008.

[12] Takanori Fukushima, Atsuko Kosaka, Yoji Ishimura, Takashi Yamamoto, Toshikazu Takigawa, Noriyuki Ishii, and Takuzo Aida. Molecular Ordering of Organic Molten Salts Triggered by Single-Walled Carbon Nanotubes. Science, 300(5628):2072-2074, June 2003.

[13] Patrice Simon and Yury Gogotsi. Materials for electrochemical capacitors. Nature Materials, 7(11):845-854, November 2008.

[14] Roger Parsons. The electrical double layer: recent experimental and theoretical developments. Chem. Rev., 90(5):813-826, July 1990.

[15] Benjamin Rotenberg and Mathieu Salanne. Structural Transitions at Ionic Liquid Interfaces. J. Phys. Chem. Lett., 6(24):4978-4985, December 2015. 
[16] Jianzhong Wu, Tao Jiang, De-en Jiang, Zhehui Jin, and Douglas Henderson. A classical density functional theory for interfacial layering of ionic liquids. Soft Matter, 7(23):11222-11231, November 2011.

[17] Martin Z Bazant, Brian D Storey, and Alexei A Kornyshev. Double Layer in Ionic Liquids: Overscreening versus Crowding. Phys. Rev. Lett., 106(4):046102, January 2011.

[18] David T Limmer. Interfacial Ordering and Accompanying Divergent Capacitance at Ionic Liquid-Metal Interfaces. Phys. Rev. Lett., 115(25):256102, December 2015 .

[19] Nir Gavish and Arik Yochelis. Theory of Phase Separation and Polarization for Pure Ionic Liquids. J. Phys. Chem. Lett., 7(7):1121-1126, April 2016.

[20] Alpha A Lee and Susan Perkin. Ion-Image Interactions and Phase Transition at Electrolyte-Metal Interfaces. J. Phys. Chem. Lett., 7(14):2753-2757, July 2016.

[21] L D Landau and E M Lifshitz. Fluid Mechanics, Second Edition: Volume 6 (Course of Theoretical Physics). Course of theoretical physics / by L. D. Landau and E. M. Lifshitz, Vol. 6. Butterworth-Heinemann, 2 edition, January 1987.

[22] G De Fabritiis, M Serrano, R Delgado-Buscalioni, and P Coveney. Fluctuating hydrodynamic modeling of fluids at the nanoscale. Phys. Rev. E, 75(2):026307, February 2007.

[23] John B Bell, Alejandro L Garcia, and Sarah A Williams. Numerical methods for the stochastic Landau-Lifshitz Navier-Stokes equations. Phys. Rev. E, 76(1): 016708, July 2007.

[24] Nikolaos K Voulgarakis and Jhih-Wei Chu. Bridging fluctuating hydrodynamics and molecular dynamics simulations of fluids. J. Chem. Phys., 130(13):134111, 2009 .

[25] Florencio Balboa Usabiaga, John B Bell, Rafael Delgado-Buscalioni, Aleksandar Donev, Thomas G Fai, Boyce E Griffith, and Charles S Peskin. Staggered Schemes for Fluctuating Hydrodynamics. http://dx.doi.org/10.1137/120864520, 10(4):1369-1408, November 2012.

[26] Barry Z Shang, Nikolaos K Voulgarakis, and Jhih-Wei Chu. Fluctuating hydrodynamics for multiscale modeling and simulation: Energy and heat transfer in molecular fluids. J. Chem. Phys., 137(4):044117, 2012.

[27] Steven Delong, Boyce E Griffith, Eric Vanden-Eijnden, and Aleksandar Donev. Temporal integrators for fluctuating hydrodynamics. Phys. Rev. E, 87(3):033302, March 2013.

[28] Nikolaos K Voulgarakis, Siddarth Satish, and Jhih-Wei Chu. Modeling the nanoscale viscoelasticity of fluids by bridging non-Markovian fluctuating hydrodynamics and molecular dynamics simulations. J. Chem. Phys., 131(23):234115, 2009. 
[29] Nikolaos K Voulgarakis, Siddarth Satish, and Jhih-Wei Chu. Modelling the viscoelasticity and thermal fluctuations of fluids at the nanoscale. Molecular Simulation, 36(7-8):552-559, August 2010.

[30] Barry Z Shang, Nikolaos K Voulgarakis, and Jhih-Wei Chu. Fluctuating hydrodynamics for multiscale simulation of inhomogeneous fluids: Mapping all-atom molecular dynamics to capillary waves. J. Chem. Phys., 135(4):044111, 2011.

[31] A Chaudhri, J B Bell, A L Garcia, and A Donev. Modeling multiphase flow using fluctuating hydrodynamics. Phys. Rev. E, 2014.

[32] Kaushik Balakrishnan, Alejandro L Garcia, Aleksandar Donev, and John B Bell. Fluctuating hydrodynamics of multispecies nonreactive mixtures. Phys. Rev. E, 89(1):013017, January 2014.

[33] Amit Kumar Bhattacharjee, Kaushik Balakrishnan, Alejandro L Garcia, John B Bell, and Aleksandar Donev. Fluctuating hydrodynamics of multi-species reactive mixtures. J. Chem. Phys., 142(22):224107, June 2015.

[34] Paul J Atzberger, Peter R Kramer, and Charles S Peskin. A stochastic immersed boundary method for fluid-structure dynamics at microscopic length scales. Journal of Computational Physics, 224(2):1255-1292, June 2007.

[35] Paul J Atzberger. Journal of Computational Physics. Journal of Computational Physics, 230(8):2821-2837, April 2011.

[36] Florencio Balboa Usabiaga, Rafael Delgado-Buscalioni, Boyce E Griffith, and Aleksandar Donev. Inertial coupling method for particles in an incompressible fluctuating fluid. COMPUTER METHODS IN APPLIED MECHANICS AND ENGINEERING, 269:139-172, February 2014.

[37] F Balboa Usabiaga, I Pagonabarraga, and R Delgado-Buscalioni. Journal of Computational Physics. Journal of Computational Physics, 235(C):701-722, February 2013.

[38] Eric E Keaveny. Fluctuating force-coupling method for simulations of colloidal suspensions. Journal of Computational Physics, 269:61-79, July 2014.

[39] B Uma, T N Swaminathan, R Radhakrishnan, D M Eckmann, and $\mathrm{P} S$ Ayyaswamy. Nanoparticle Brownian motion and hydrodynamic interactions in the presence of flow fields. Phys. Fluids, 23(7):073602, 2011.

[40] Mordechai Bixon and Robert Zwanzig. Boltzmann-Langevin Equation and Hydrodynamic Fluctuations. Phys. Rev., 187(1):267-272, November 1969.

[41] Ronald Forrest Fox and George E Uhlenbeck. Contributions to Non-Equilibrium Thermodynamics. I. Theory of Hydrodynamical Fluctuations. Physics of Fluids (1958-1988), 13(8):1893-1902, August 1970.

[42] Rebecca Honeycutt. Stochastic Runge-Kutta algorithms. I. White noise. Phys. Rev. A, 45(2):600-603, January 1992. 
[43] William H Press, Saul A Teukolsky, William T Vetterling, and Brian P Flannery. Numerical Recipes in FORTRAN; The Art of Scientific Computing. Cambridge University Press, New York, NY, USA, 2nd edition, 1993.

[44] Non-Markovian Fluctuation Hydrodynamics for ionic liquids under external electrostatic field. in preperation, 2017.

[45] Adrian J Carmichael, Christopher Hardacre, John D Holdbrey, Mark Nieuwenhuyzen, and Kenneth R Seddon. Molecular layering and local order in thin films of 1-alkyl-3-methylimidazolium ionic liquids using X-ray reflectivity. Molecular Physics, 99(10):795-800, November 2009.

[46] Judith Hoth, Florian Hausen, Martin H Müser, and Roland Bennewitz. Force microscopy of layering and friction in an ionic liquid. J. Phys.: Condens. Matter, 26(28):284110, July 2014. 


\section{FIGURES}

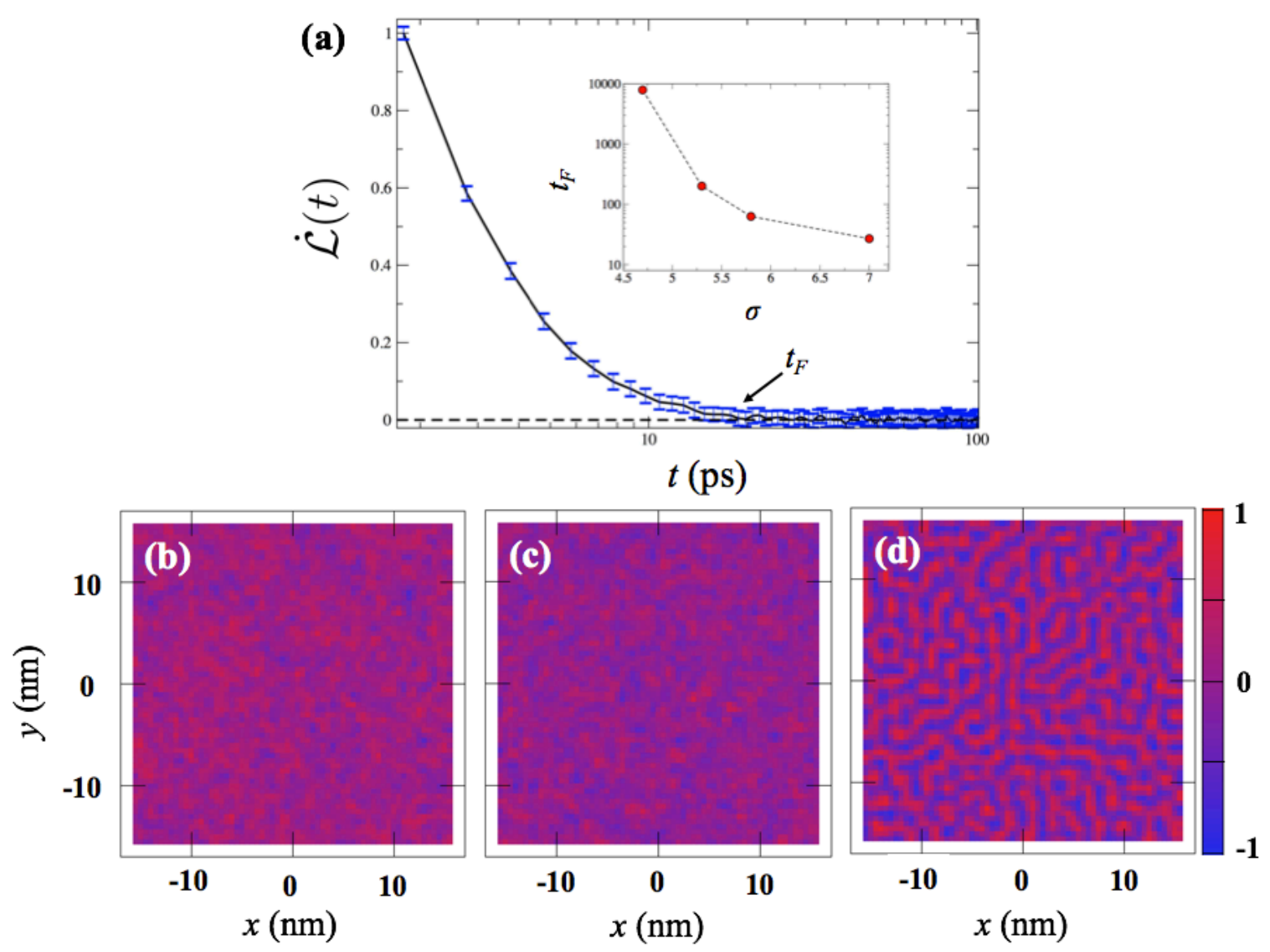

Figure 1: (a) Norailized time derivative of the total free energy, i.e., $\dot{\mathcal{L}}(t)=\dot{F}(t) / \dot{F}(0)$, as a function of time. This profile corresponds to the average of 100 independent simulations. Error bars correspond to standard deviations. The equalibration time, $t_{F}$, is defined as the time required for $\dot{\mathcal{L}}(t)$ to reach a zero. In practice, $\dot{\mathcal{L}}(t) \leq 0.01$ for $t \geq t^{*}$. Second row shows the density plots of a) mass density, b) momentum density, and c) mass fraction difference at $t=t_{F}+5$ ns. i.e., $t \gg t_{F}$. The order paramter is $\sigma=7$. 

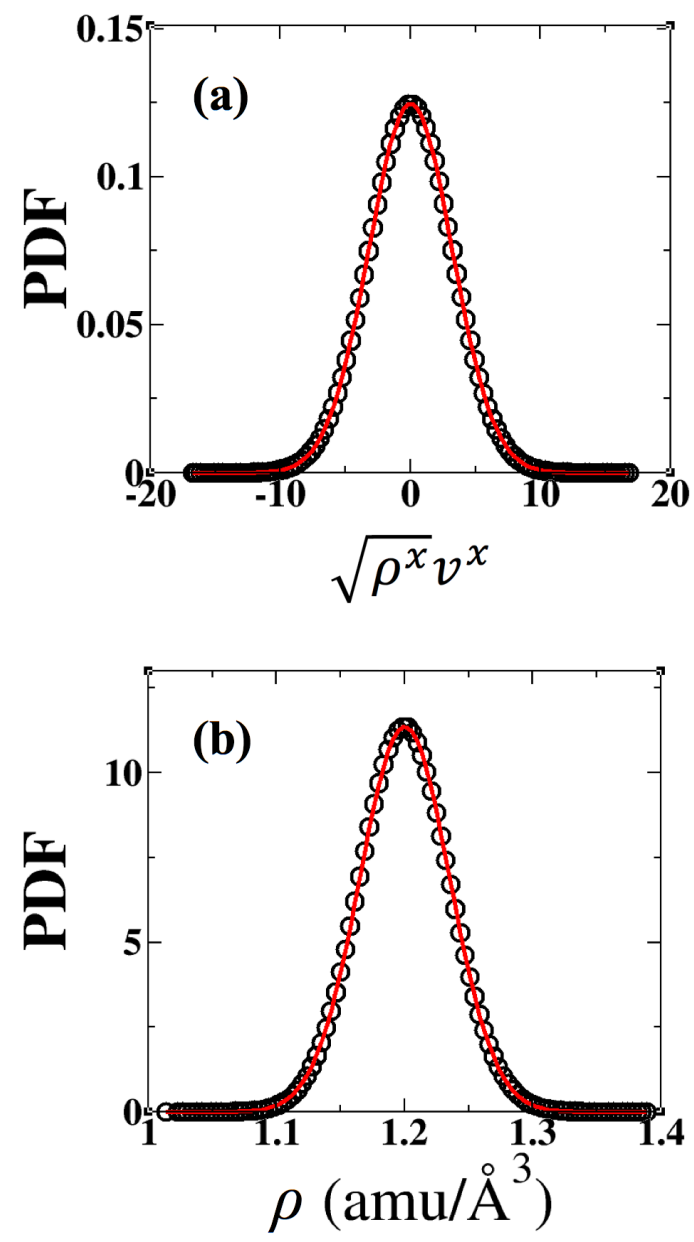

Figure 2: Probability density functions (PDF) of a) $\sqrt{\rho^{x}} v^{x}$ and b) mass density for $\sigma=7$. The solid lines correspond to the best fit curves of functions (22) and (23), respectivily. 

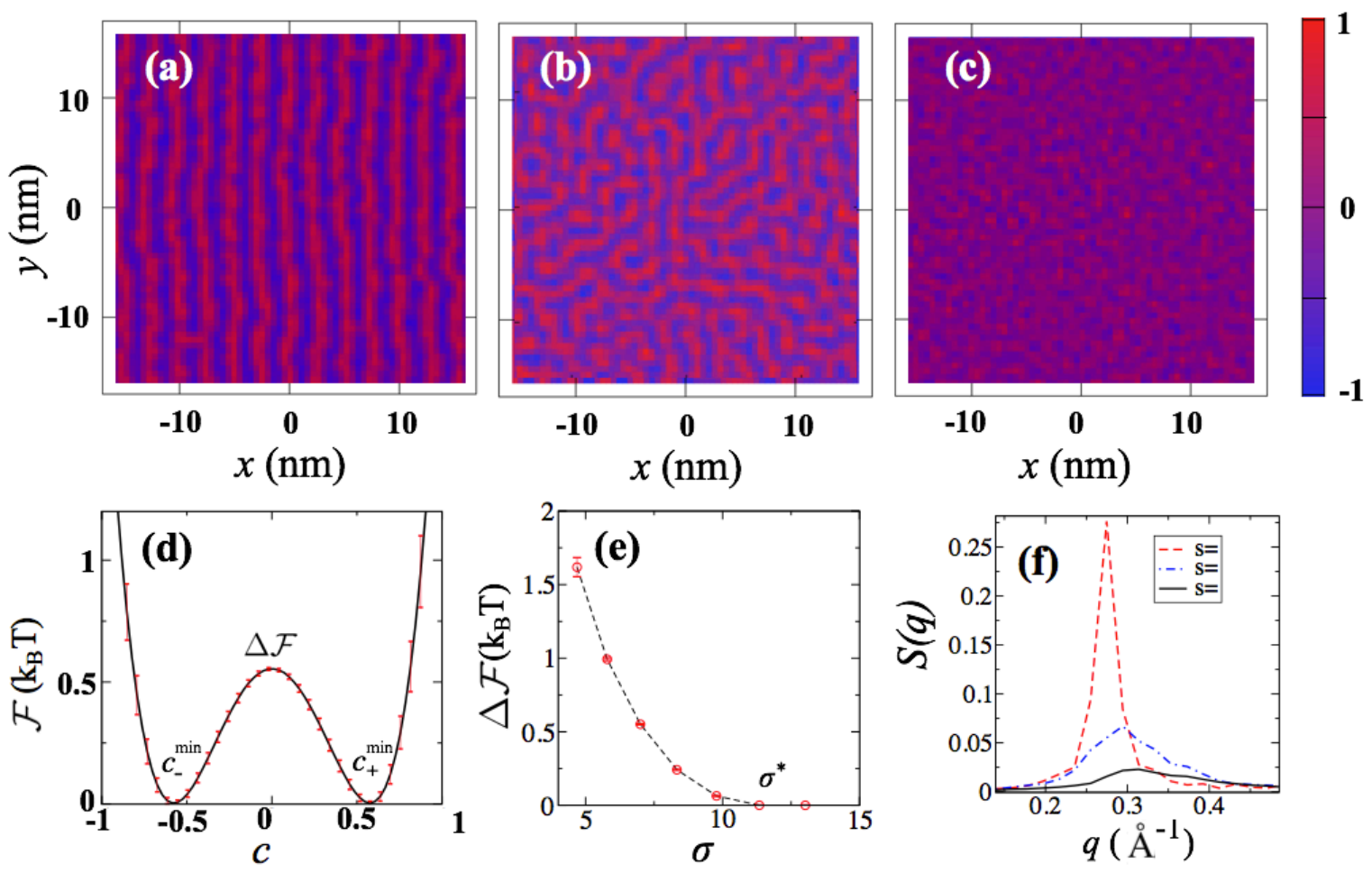

Figure 3: Instanteneous density plots of $c$ at time $t=t_{e q}+5$ ns for a) $\sigma=0.23$, b) $\sigma=3.7$, and c) $\sigma=23.1$. (d) Effective free energy as a function of $c$ for $\sigma=7$. The two minima of the double well are denoted by $c_{-}^{\min }$ and $c_{+}^{\min }$, The free energy barrier is represented by $\Delta \mathcal{F}$. (e) $\Delta \mathcal{F}$ as a function of the order parameter $\sigma$. The energy barrier vanishes for $\sigma^{*} \approx 10$. (f) Nonnormalized power spectra of $c$ as a function of $q$ for $\sigma=0.23$ (dashed line), $\sigma=3.7$ (dashed-dot line), and $\sigma=23.1$ (solid line). Error bars correspond to standard deviations. 


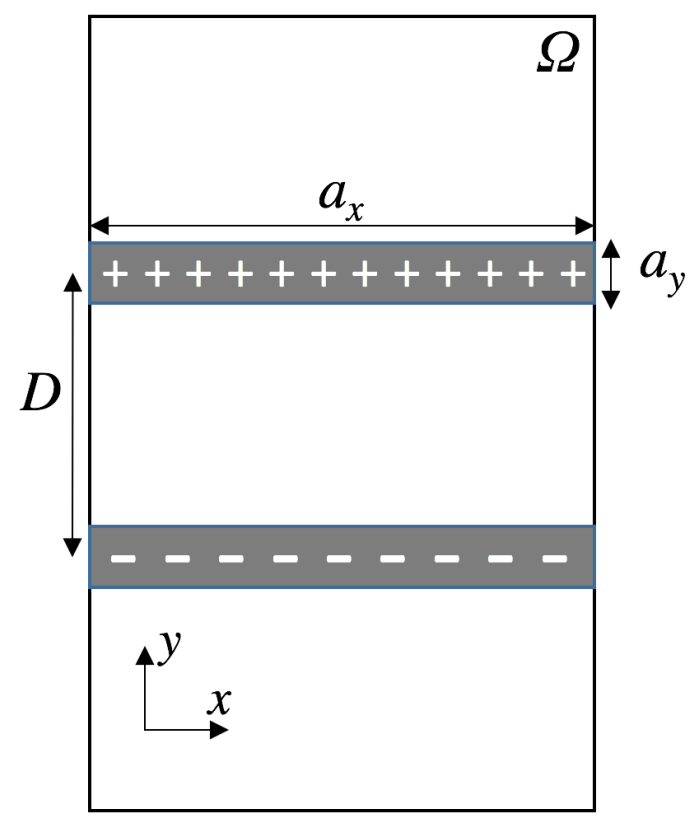

Figure 4: Schematic representation of the two oppositely charged electrodes immersed in an ionic liquid. The dimensions of the two electrodes are $a_{x}=16$ Åand $a_{y}=16 \AA$, while the interdistance is $D=100 \AA$. 

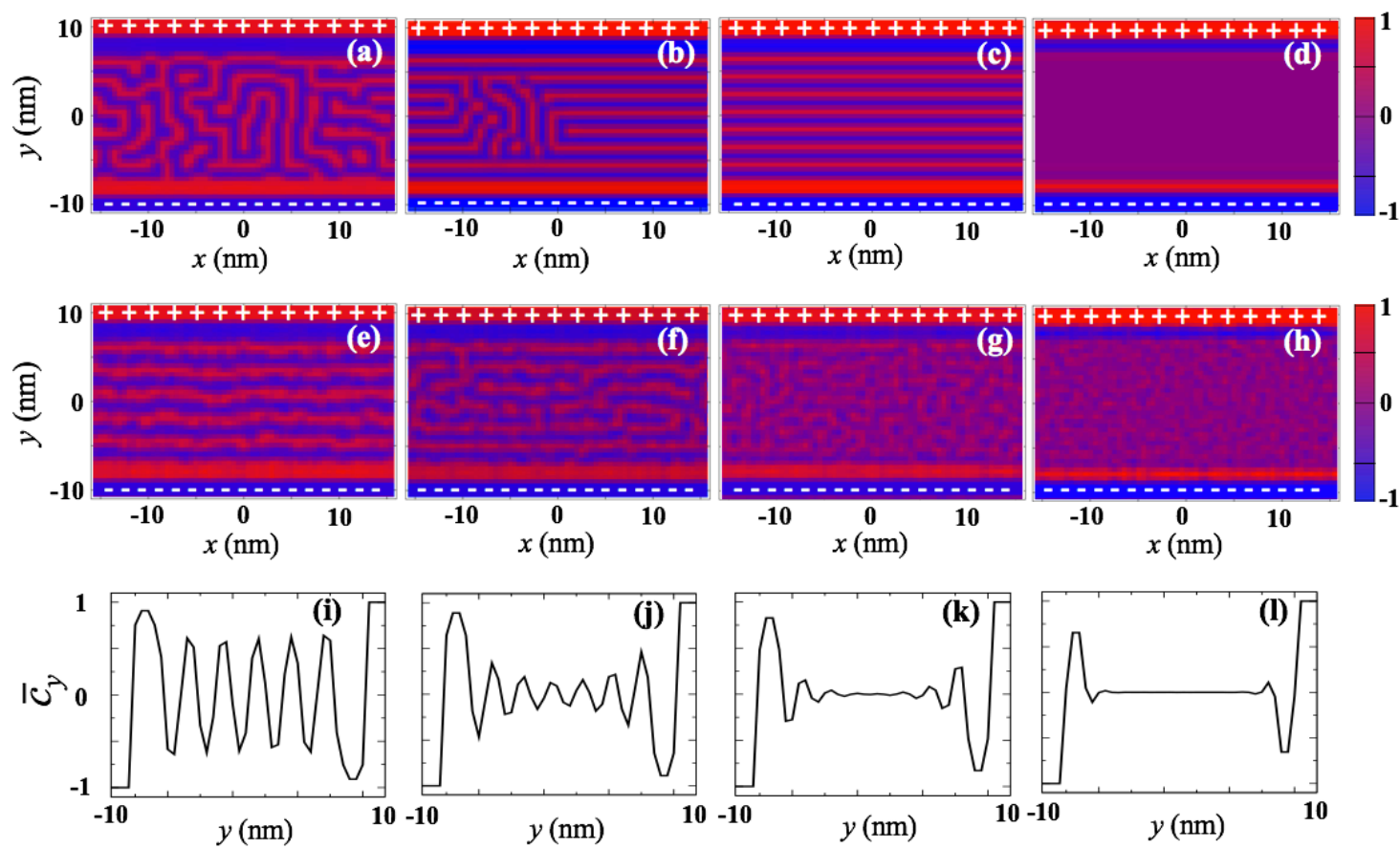

Figure 5: First row: Deterministic simulations for (a) $\sigma=0.9$, (b) $\sigma=8.3$, (c) $\sigma=9.8$, (d) $\sigma=21.1$. Snapshots correspond to density plot of mass fraction difference, $c$. Second row: Fluctuating hydrodynamics simulations for (a) $\sigma=0.9$, (b) $\sigma=5.8$, (c) $\sigma=9.8$, (d) $\sigma=21.1$.. Snapshots correspond to density plot of $c$. Third row shows the corresponding statistical average of c over $x$-axis as a function of $y$ for the FHD simulations shown in the second row. 